\title{
DEGREE REDUCTION OF BÉZIER CURVES AND ITS ERROR ANALYSIS
}

\author{
YUNBEOM PARK and U JIN CHOI ${ }^{1}$
}

(Received 16 July 1993; revised 4 October 1993)

\begin{abstract}
The error analysis of an algorithm for generating an approximation of degree $n-1$ to an $n$th degree Bézier curve is presented. The algorithm is based on observations of the geometric properties of Bezier curves which allow the development of detailed error analysis. By combining subdivision with a degree reduction algorithm, a piecewise approximation can be generated, which is within some preset error tolerance of the original curve. The number of subdivisions required can be determined a priori and a piecewise approximation of degree $m$ can be generated by iterating the scheme.
\end{abstract}

\section{Introduction}

Degree reduction of Bézier curves and surfaces is a process that amounts to approximating a polynomial of degree $n$ by one of degree $m<n$. In most methods, $m=n-1$ so that to obtain an approximation of a lower degree the scheme must be used recursively. This way a series of approximations is generated, each obtained by reducing the polynomial degree by one at each stage.

One of the main uses of a degree reduction algorithm is to generate a piecewise linear approximation to a prescribed curve or surface. These piecewise linear approximations are important because of their use in rendering curve-curve, curvesurface and surface-surface intersection calculations. To generate a piecewise linear approximation to within a specified error tolerance, one must combine a subdivision algorithm with degree reduction. For Bézier curves and surfaces such algorithms are easy to implement. Subdivision/degree reduction in two and three variables has been investigated by Petersen [7] in the context of intersection problems.

The degree reduction is relevant also to other application areas such as CAGD (Computer Aided Geometric Design). The application of the algorithm is transferring

\footnotetext{
${ }^{1}$ Department of Mathematics, Korea Advanced Institute of Science and Technology, Gu-sung Dong, Yu-sung Gu, Taejon, 305-701, Republic of Korea.

(C) Australian Mathematical Society, 1995, Serial-fee code 0334-2700/95
} 
data from one geometric modelling system to another. Often a curve of high degree must be approximated by a number of curves of lower degree, due to the limitation on the maximum polynomial degree that certain systems can store and work with. Such situations often arise, in practice, and some work in this area has been done. See, for instance, Dannenberg and Nowacki [2] or Hoscheck [6].

The use of Bézier curves finds much application in general description of curves and surfaces and provides the mathematical basis for many CAD (Computer Aided Design) systems. Boehm, Farin and Kahmann [1] summarize the basic theory of such curves and provide many relevant references. The Bézier representation uses Bernstein polynomials as basis functions for the linear space of polynomials. In terms of the Bernstein polynomials of degree $n$,

$$
B_{i}^{n}(t)=\left(\begin{array}{c}
n \\
i
\end{array}\right)(1-t)^{n-i} t^{i}, \quad 0 \leq t \leq 1, \quad i=0, \ldots, n,
$$

a parametric polynomial curve of degree $n(n>0)$ in the plane, can be expressed as

$$
\sum_{i=0}^{n} b_{i} B_{i}^{n}(t), \quad b_{i} \in R^{2}
$$

(Boehm et al., [1]). The points $b_{i}, i=0, \ldots, n$, are called the control points for the polynomial and the polygon formed joining successive control points is the control polygon. Notice that $b_{0}$ and $b_{n}$ are the end points of the curve (corresponding to $t=0$ and $t=1$ ). Moreover, the vectors $b_{0}-b_{1}$ and $b_{n}-b_{n-1}$ define the tangents to the curve at the two end points respectively. Figure 1 shows an example of a parametric Bézier curve of degree 5 and its control polygon.

In the second section of the paper, a method for approximation is presented. Although a similar method has already been considered by Farin [3], he did not analyze the error. Hersch [5] developed a degree reduction algorithm for font conversion without error analysis. Sederberg and Kakimoto [8] consider the problem for approximating rational curves using polynomial curves. Watkins and Worsey [9] developed an algebraic method for approximation using Tchebycheff polynomials. The approach taken here is different. The authors aim to develop a geometric method for generating lower degree approximations which, in the uniform norm, are within some preset error tolerance of the prescribed Bézier curve. This means that the scheme for approximation must be combined with a subdivision algorithm.

This question is addressed in the third section. The techniques used in the approximation algorithm admit a detailed error analysis for the method. These results are presented and used to determine, a priori, the number of subdivisions that are needed for the approximation to be within the error tolerance that is imposed. The original curve is subdivided and each segment of it is approximated separately. 


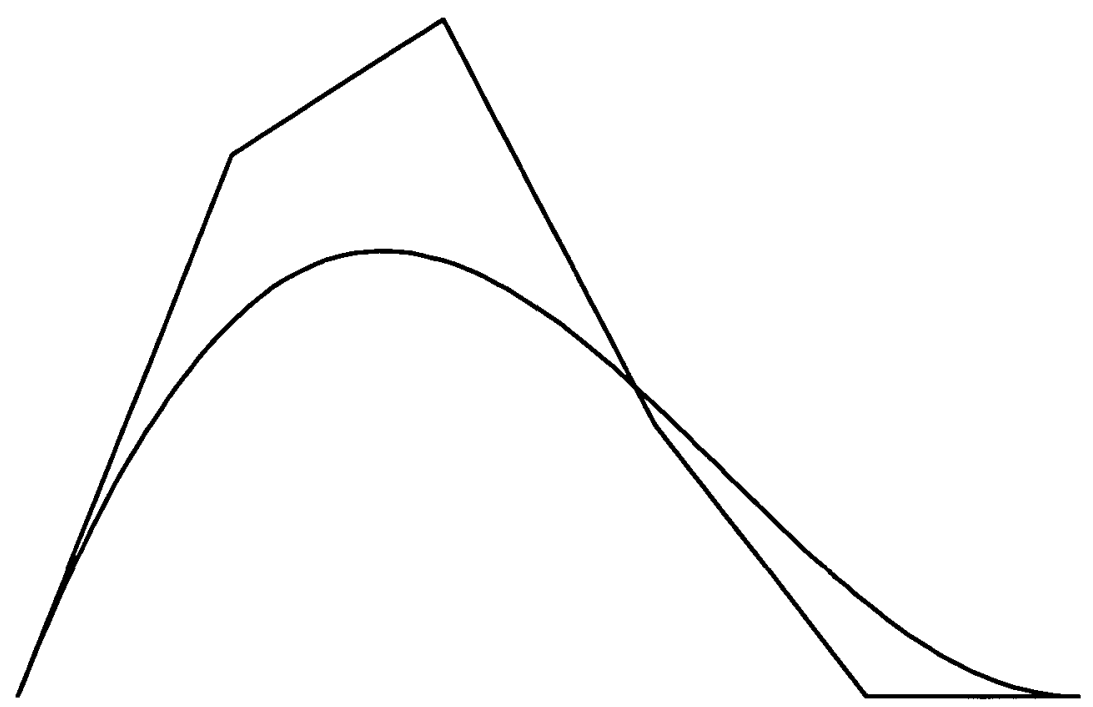

FIGURE 1. Parametric Bézier curve of degree 5 and its control polygon.

The method described below has an obvious extension to higher dimensions to deal with the problem of approximating tensor product surfaces. The problem is simply decomposed into a series of univariate problems.

\section{Degree reduction}

Degree elevation can be viewed as a process that introduces redundancy: a curve is described by more information than is actually necessary. The inverse process might seem more interesting: can we reduce possible redundancy in a curve representation? More specifically, can we write a given curve of degree $n$ as one of degree $n-1$ ? We shall call that process degree reduction.

In general, exact degree reduction is not possible. Degree reduction, therefore, can only be viewed as a method to approximate a given curve by one of lower degree. Our problem can now be stated as follows: given a Bézier curve with control vertices $b_{i}, i=0, \ldots, n$, can we find a Bézier curve with control vertices $q_{i}, i=0, \ldots, n-1$, that approximates the first curve?

Let us now pretend that the $b_{i}$ were obtained from the $q_{i}$ by the process of degree 
elevation. Then they would be related by ([4])

$$
\begin{aligned}
b_{0} & =q_{0}, \\
b_{i} & =\frac{i}{n} q_{i-1}+\frac{n-i}{n} q_{i}, \quad i=1, \ldots, n-1, \\
b_{n} & =q_{n-1} .
\end{aligned}
$$

This equation can be used to derive two recursive formulas for the generation of the $q_{i}$ from $b_{i}$ :

$$
\begin{aligned}
q_{0} & =b_{0}, \\
q_{i} & =\frac{n}{n-i} b_{i}-\frac{i}{n-i} q_{i-1}, \quad i=1, \ldots, n-1, \quad \text { and } \\
q_{n-1} & =b_{n}, \\
q_{i-1} & =\frac{n}{i} b_{i}-\frac{n-i}{i} q_{i}, \quad i=n-1, \ldots, 1 .
\end{aligned}
$$

Let $m=\left[\frac{n}{2}\right]$, where $[\cdot]$ is the floor function. From these formulae, to find the approximation, we define auxiliary points $q_{i}^{l}$ and $q_{i}^{r}$ as follows:

$$
\begin{aligned}
& q_{0}^{l}=b_{0}, \\
& q_{i}^{l}=\frac{n}{n-i} b_{i}-\frac{i}{n-i} q_{i-1}^{l}, \quad \begin{cases}i=1, \ldots, m & \text { if } n \text { is odd } \\
i=1, \ldots, m-1 & \text { if } n \text { is even }\end{cases}
\end{aligned}
$$

and

$$
\begin{aligned}
& q_{n-1}^{r}=b_{n}, \\
& q_{i-1}^{r}=\frac{n}{i} b_{i}-\frac{n-i}{i} q_{i}^{r}, \quad i=n-1, \ldots, m+1 .
\end{aligned}
$$

Then we can rewrite the Bézier curve $b^{n}$ as

$$
\begin{aligned}
b^{n}(t) & =\sum_{i=0}^{n} b_{i} B_{i}^{n}(t) \\
& =\sum_{i=0}^{m-1} q_{i}^{l} B_{i}^{n-1}(t)+\left[q_{m}^{l}(1-t)+q_{m}^{r} t\right] B_{m}^{n-1}(t)+\sum_{i=m+1}^{n-1} q_{i}^{r} B_{i}^{n-1}(t)
\end{aligned}
$$

for odd $n$ and

$$
\begin{aligned}
b^{n}(t)= & \sum_{i=0}^{n} b_{i} B_{i}^{n}(t) \\
= & \sum_{i=0}^{m-2} q_{i}^{l} B_{i}^{n-1}(t)+\left[q_{m-1}^{l}(1-t)+b_{m} t\right] B_{m-1}^{n-1}(t) \\
& +\left[b_{m}(1-t)+q_{m}^{r} t\right] B_{m}^{n-1}(t)+\sum_{i=m+1}^{n-1} q_{i}^{r} B_{i}^{n-1}(t)
\end{aligned}
$$


for even $n$.

The reduction curve $q^{n-1}(t)=\sum_{i=0}^{n-1} q_{i} B_{i}^{n-1}(t)$ of degree $n-1$ can now be defined as follows:

$$
q_{i}= \begin{cases}q_{i}^{l} & \text { for } i=0,1, \ldots, m-1 \\ q_{m} \in\left\{q_{m}^{l}(1-s)+q_{m}^{r} s, 0 \leq s \leq 1\right\} & \\ q_{i}^{r} & \text { for } i=m+1, \ldots, n-1,\end{cases}
$$

for odd $n$ and

$$
q_{i}= \begin{cases}q_{i}^{l} & \text { for } i=0,1, \ldots, m-2 \\ q_{m-1} \in\left\{q_{m-1}^{l}(1-s)+b_{m} s, 0 \leq s \leq 1\right\} & \\ q_{m} \in\left\{b_{m} s+q_{m}^{r}(1-s), 0 \leq s \leq 1\right\} & \\ q_{i}^{r} & \text { for } i=m+1, \ldots, n-1,\end{cases}
$$

for even $n$.

The techniques in the approximation algorithm admit a detailed error analysis for the method. For first error analysis, we use the parametric Euclidean uniform norm III $\mid$ II defined by

$$
\|\mid b\| \|=\left\{\|x\|^{2}+\|y\|^{2}\right\}^{\frac{1}{2}}
$$

for a parametric curve $b$, where $\|\cdot\|$ is the uniform norm. Let $e(t)=b^{n}(t)-q^{n-1}(t)=$ $\left(e^{x}(t), e^{y}(t)\right)$ be the parametric difference of the two curves and set $s=\frac{1}{2}$ for odd $n$ and $s=0$ for even $n$, then

$$
e(t)=\left\{\begin{array}{cl}
\frac{1}{2}\left(\begin{array}{c}
n-1 \\
m
\end{array}\right)(1-2 t)(1-t)^{n-m-1} t^{m}\left(q_{m}^{l}-q_{m}^{r}\right) & \text { if } n \text { is odd } \\
2\left(\begin{array}{c}
n-1 \\
m
\end{array}\right)(1-t)^{n-m} t^{m}\left(b_{m}-\frac{q_{m-1}^{l}+q_{m}^{r}}{2}\right) & \text { if } n \text { is even. }
\end{array}\right.
$$

For computation of $\||e|\|$, we first consider $\left\|e^{x}\right\|$ and $n$ is odd.

$$
\begin{aligned}
\left\|e^{x}\right\| & =\left|q_{m}^{l x}-q_{m}^{r x}\right| \frac{1}{2}\left(\begin{array}{c}
n-1 \\
m
\end{array}\right) \max _{0 \leq r \leq 1}\left|(1-2 t)(1-t)^{n-m-1} t^{m}\right| \\
& \leq \frac{1}{2}\left(\begin{array}{c}
n-1 \\
m
\end{array}\right) \frac{1}{\sqrt{n}}\left(\frac{1}{4}-\frac{1}{4 n}\right)^{m}\left|q_{m}^{l^{x}}-q_{m}^{r x}\right| .
\end{aligned}
$$

By applying similar calculations, we obtain the same form for $\left\|e^{y}\right\|$.

For even $n$,

$$
\begin{aligned}
\left\|e^{x}\right\| & =\left|b_{m}{ }^{x}-\frac{q_{m-1}^{l}{ }^{x}+q_{m}^{r x}}{2}\right| 2\left(\begin{array}{c}
n-1 \\
m
\end{array}\right) \max _{0 \leq t \leq 1}\left|(1-t)^{m} t^{m}\right| \\
& \leq 2\left(\begin{array}{c}
n-1 \\
m
\end{array}\right)\left(\frac{1}{2}\right)^{n}\left|b_{m}{ }^{x}-\frac{q_{m_{1}}^{l^{x}}+q_{m}^{r x}}{2}\right| .
\end{aligned}
$$




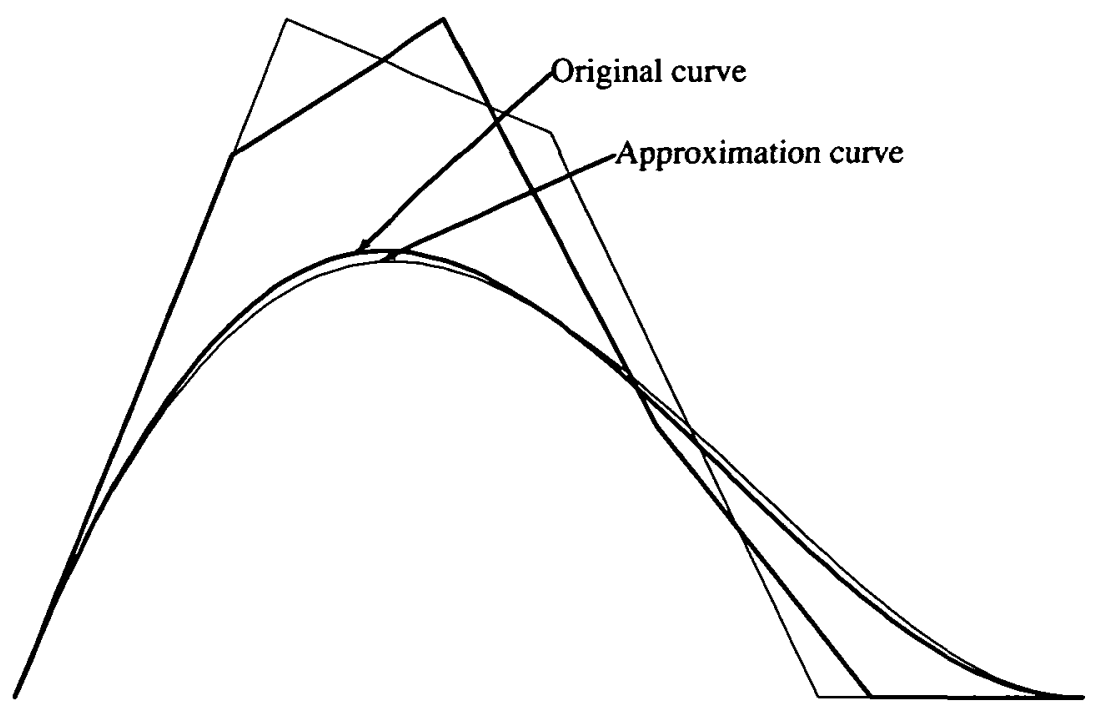

FIGURE 2. Degree reduction on a Bézier curve of degree 5.

We obtain the same form for $\left\|e^{y}\right\|$ by similar calculations.

Combining these results, we obtain the following theorem:

THEOREM 1. The error of the suggested degree reduction algorithm is

$$
\|\mid\| \| \leq \frac{1}{2}\left(\begin{array}{c}
n-1 \\
m
\end{array}\right) \frac{1}{\sqrt{n}}\left(\frac{1}{4}-\frac{1}{4 n}\right)^{m} d\left(q_{m}^{l}, q_{m}^{r}\right)
$$

for odd $n$ and

$$
\||||| \leq 2\left(\begin{array}{c}
n-1 \\
m
\end{array}\right)\left(\frac{1}{2}\right)^{n} d\left(b_{m}, \frac{q_{m-1}^{l}+q_{m}^{r}}{2}\right)
$$

for even n, where d is Euclidean distance.

The replacement curve is to approximate the original curve in the sense that the two should lie within some specified tolerance $(\epsilon)$ of each other. We thus only check the distance of two points $q_{m}^{l}$ and $q_{m}^{r}$ for odd $n, b_{m}$ and $\frac{q_{m-1}^{\prime}+q_{m}^{r}}{2}$ for even $n$. Figure 2 and Figure 3 show the results of applying the degree reduction method presented in this section. 


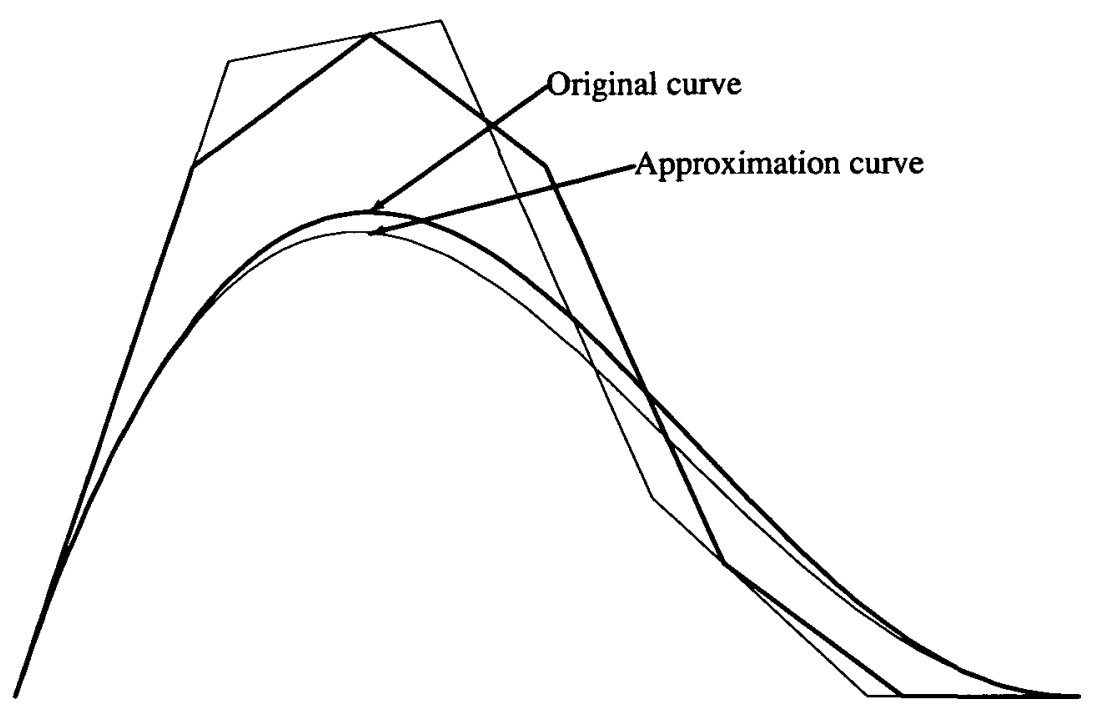

FIGURE 3. Degree reduction on a Bézier curve of degree 6.

\section{Subdivision/degree reduction}

In this section, we give an error analysis of the subdivision and approximation process. Our results show when and how often one should subdivide and/or attempt degree reduction. In particular, it is shown that for a curve $b^{n}$ of degree $n$, it is possible to determine a priori how many times one must subdivide $b^{n}$ before it can be approximated to within a given tolerance by curves of degree one less. Thus it is not necessary to attempt approximation after each subdivision.

A Bézier curve $b^{n}$ is usually defined over the interval $[0,1]$, but it can also be defined over any interval $[0, c]$. The part of the curve that corresponds to $[0, c]$ can also be defined by a Bézier polygon, as illustrated in Figure 4. Finding this Bézier polygon is referred to as subdivision of the Bézier curve. Recursive subdivision of Bézier curves is based on de Casteljau's theorem ([4]).

Let us denote the Bézier polygon corresponding to the interval $[0, c]$ by $c_{0}, \ldots, c_{n}$ it defines a Bézier curve $c^{n}$ (which is part of the same curve as $b^{n}$ is, of course). We have the following subdivision formula for Bézier curves:

$$
c_{j}=b_{0}^{j}(c)
$$



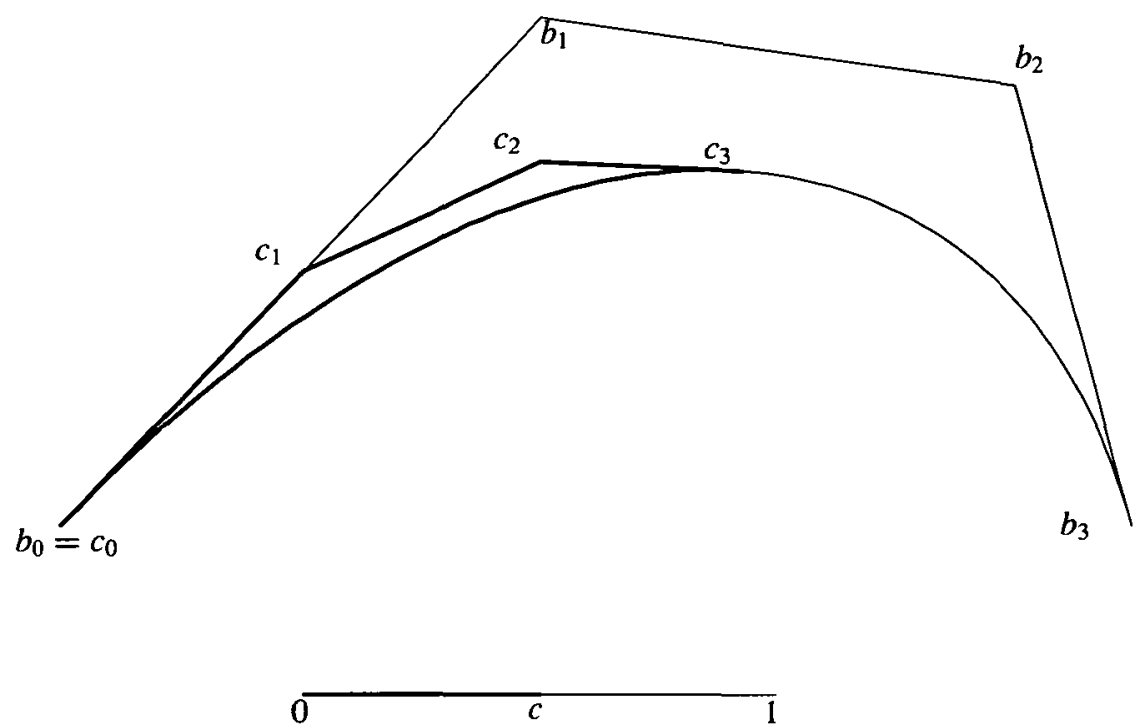

FIGURE 4. Subdivision of a Bézier curve.

where $b_{0}^{j}(c)$ is recursively defined by

$$
b_{i}^{j}(c)=(1-c) b_{i}^{j-1}(c)+c b_{i+1}^{j-1}(c) \quad\left\{\begin{array}{l}
j=1, \ldots, n \\
i=0, \ldots, n-j
\end{array}\right.
$$

and $b_{i}^{0}(c)=b_{i}$. The control vertices corresponding to $[c, 1]$ are given by the $b_{n-j}^{j}$. From the formula (3.1), we easily obtain the following lemma:

LEMMA 2. Another formula for $c_{j}$ is

$$
c_{j}= \begin{cases}\sum_{k=0}^{j} B_{k}^{j}(c) b_{k} & \text { for }[0, c], \\ \sum_{k=0}^{j} B_{k}^{j}(c) b_{n-j+k} & \text { for }[c, 1] .\end{cases}
$$

In the previous section, we mentioned that one only needs to check the distance of two points. If the distance $d$ is greater than $\epsilon / \alpha_{n}$, one attempts to subdivide the given $n$th degree Bézier curve and construct the auxiliary points for each subdivided curve 
segment, where $\alpha_{n}$ is

$$
\begin{array}{ll}
\frac{1}{2}\left(\begin{array}{c}
n-1 \\
m
\end{array}\right) \frac{1}{\sqrt{n}}\left(\frac{1}{4}-\frac{1}{4 n}\right)^{m} & \text { for odd } n \\
2\left(\begin{array}{c}
n-1 \\
m
\end{array}\right)\left(\frac{1}{2}\right)^{n} & \text { for even } n .
\end{array}
$$

If all distances of two new points is less than or equal to $\epsilon / \alpha_{n}$, replace the given Bezier curve by the Bézier curve of degree $n-1$. If not, subdivide until all distances of the two new points are less than or equal to $\epsilon / \alpha_{n}$.

Let $e^{(k)}$ be the parametric difference of the original curve and the replacement curve at the $k$ th step. Let us denote the control polygon of the approximation corresponding to the interval $[0, c]$ by $r_{0}, \ldots, r_{n-1}$ and auxiliary points $r_{i}^{l}$ and $r_{i}^{r}$. In the following two subsections, we give the error analysis of the subdivision and degree reduction process.

3.1. For odd degree $n$ The matrix form of the recursive formula for $q_{i}^{l}$ is $M^{l} Q^{l}=B^{l}$, where the matrix $M^{l}$ is

$$
\left(\begin{array}{ccccccc}
\frac{n}{n} & 0 & 0 & \ldots & 0 & 0 & 0 \\
\frac{1}{n} & \frac{n-1}{n} & 0 & \ldots & 0 & 0 & 0 \\
0 & \frac{2}{n} & \frac{n-2}{n} & \cdots & 0 & 0 & 0 \\
\vdots & \vdots & \vdots & & \vdots & \vdots & \vdots \\
0 & 0 & 0 & \ldots & \frac{m-1}{n} & \frac{n-(m-1)}{n} & 0 \\
0 & 0 & 0 & \ldots & 0 & \frac{m}{n} & \frac{n-m}{n}
\end{array}\right)
$$

and $Q^{l}=\left(q_{0}^{l}, q_{1}^{l}, \ldots, q_{m}^{l}\right)^{\top}$ and $B^{l}=\left(b_{0}, b_{1}, \ldots, b_{m}\right)^{\top}$. The matrix form for $q_{i}^{r}$ is $M^{r} Q^{r}=B^{r}$, where $M^{r}=M^{l}$ and $Q^{r}=\left(q_{n-1}^{r}, q_{n-2}^{r}, \ldots, q_{m+1}^{r}, q_{m}^{r}\right)^{\top}$ and $B^{r}=$ $\left(b_{n}, b_{n-1}, \ldots, b_{m+2}, b_{m+1}\right)^{\top}$. We obtain the following formulae for $q_{m}^{l}$ and $q_{m}^{r}$ from these systems by Gaussian elimination:

$$
\begin{aligned}
& q_{m}^{l}=\frac{1}{\left(\begin{array}{c}
n-1 \\
m
\end{array}\right)} \sum_{i=0}^{m}(-1)^{n-m+i+1}\left(\begin{array}{l}
n \\
i
\end{array}\right) b_{i}, \\
& q_{m}^{r}=\frac{1}{\left(\begin{array}{c}
n-1 \\
m
\end{array}\right)} \sum_{i=m+1}^{n}(-1)^{n-m+i}\left(\begin{array}{l}
n \\
i
\end{array}\right) b_{i} .
\end{aligned}
$$

Thus we obtain

$$
q_{m}^{l}-q_{m}^{r}=\frac{1}{\left(\begin{array}{c}
n-1 \\
m
\end{array}\right)} \sum_{i=0}^{n}(-1)^{n-m+i+1}\left(\begin{array}{l}
n \\
i
\end{array}\right) b_{i}
$$


that is,

$$
\begin{aligned}
& \left|q_{m}^{l^{x}}-q_{m}^{r x}\right|=\frac{1}{\left(\begin{array}{c}
n-1 \\
m
\end{array}\right)}\left|\sum_{i=0}^{n}(-1)^{i}\left(\begin{array}{l}
n \\
i
\end{array}\right) b_{i}{ }^{x}\right| \\
& \left|q_{m}^{l^{y}}-q_{m}^{r y}\right|=\frac{1}{\left(\begin{array}{c}
n-1 \\
m
\end{array}\right)}\left|\sum_{i=0}^{n}(-1)^{i}\left(\begin{array}{l}
n \\
i
\end{array}\right) b_{i}{ }^{y}\right|
\end{aligned}
$$

To compute $d\left(r_{m}^{l}, r_{m}^{r}\right)$, we first consider $\left|r_{m}^{l x}-r_{m}^{r x}\right|$. From (3.4),

$$
\left|r_{m}^{l^{x}}-r_{m}^{r x}\right|=\frac{1}{\left(\begin{array}{c}
n-1 \\
m
\end{array}\right)}\left|\sum_{i=0}^{n}(-1)^{i}\left(\begin{array}{l}
n \\
i
\end{array}\right) c_{i}^{x}\right| .
$$

Then, from this formula and the subdivision formula (3.2) for $c_{i}$ at $c=\frac{1}{2}$,

$$
\begin{aligned}
\left|r_{m}^{l x}-r_{m}^{r x}\right| & =\frac{1}{\left(\begin{array}{c}
n-1 \\
m
\end{array}\right)}\left|\sum_{i=0}^{n}(-1)^{i} B_{i}^{n}\left(\frac{1}{2}\right) b_{i}{ }^{x}\right| \\
& =\frac{\left(\frac{1}{2}\right)^{n}}{\left(\begin{array}{c}
n-1 \\
m
\end{array}\right)}\left|\sum_{i=0}^{n}(-1)^{i}\left(\begin{array}{c}
n \\
i
\end{array}\right) b_{i}{ }^{x}\right|,
\end{aligned}
$$

that is,

$$
\left|r_{m}^{l x}-r_{m}^{r x}\right|=\left(\frac{1}{2}\right)^{n}\left|q_{m}^{l x}-q_{m}^{r x}\right| .
$$

By similar calculations, we obtain the same form for $\left|r_{m}^{l}{ }^{x}-r_{m}^{r x}\right|$. Combining these results, we obtain

$$
d\left(r_{m}^{l}, r_{l}^{r}\right)=\left(\frac{1}{2}\right)^{n} d\left(q_{m}^{l}, q_{l}^{r}\right) .
$$

Thus the approximation error $\left\|\left|e^{(1)}\right|\right\|$ after the first subdivision is

$$
\left\|\left|e^{(1)}\right|\right\| \leq\left(\frac{1}{2}\right)^{n} \alpha_{n} d\left(q_{m}^{l}, q_{l}^{r}\right) .
$$

By induction on $k$, we reach the theorem:

THEOREM 3. The approximation error $\left\|e^{(k)} \mid\right\|$ after the $k$ th subdivision is

$$
\left\|\left|e^{(k)}\right|\right\| \leq\left(\frac{1}{2}\right)^{n k} \alpha_{n} d\left(q_{m}^{l}, q_{l}^{r}\right)
$$

for odd $n$, where $\alpha_{n}$ is

$$
\frac{1}{2}\left(\begin{array}{c}
n-1 \\
m
\end{array}\right) \frac{1}{\sqrt{n}}\left(\frac{1}{4}-\frac{1}{4 n}\right)^{m}
$$




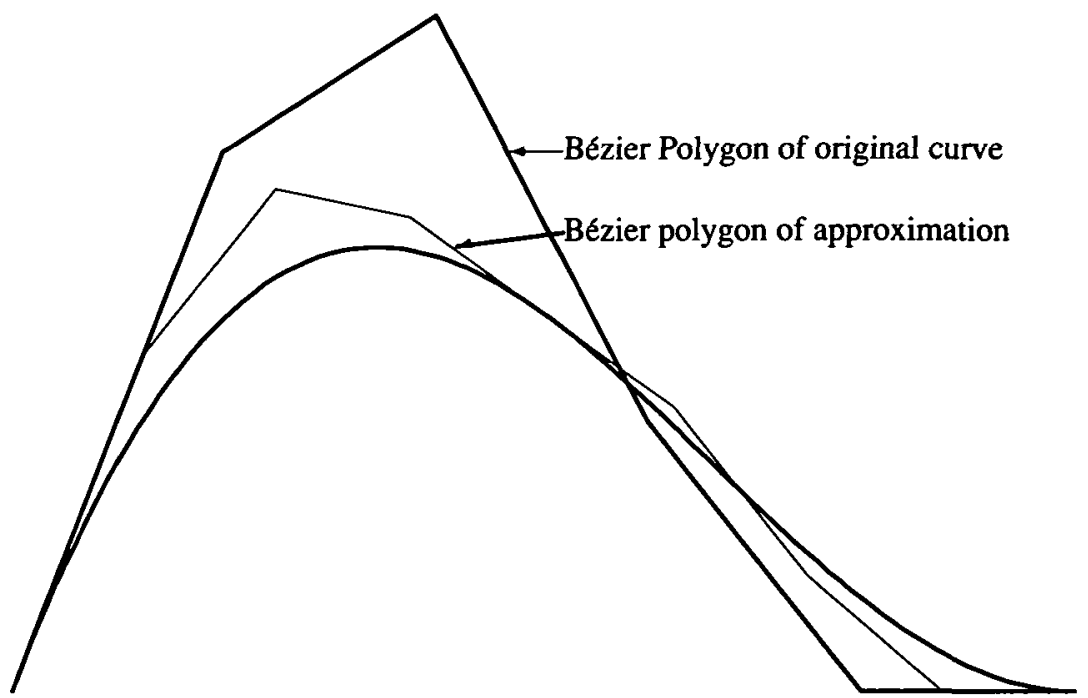

FIGURE 5. Degree reduction with 1-fold subdivision of a curve of degree 5.

Thus to obtain a piecewise Bézier approximation curve of degree $n-1$ to a given Bézier curve of degree $n$ to within a tolerance $\epsilon$, the number of subdivisions $k$ required is

$$
k \geq \frac{1}{n} \log _{2} \frac{\alpha_{n} d\left(q_{m}^{l}, q_{m}^{r}\right)}{\epsilon} .
$$

Figure 5 shows the result of applying the subdivision and degree reduction for an odd degree curve.

3.2. For even degree $n$ The matrix form of the recursive formula for $q_{i}^{l}$ is $M^{l} Q^{l}=$ $B^{l}$, where the matrix $M^{l}$ is

$$
\left(\begin{array}{ccccccc}
\frac{n}{n} & 0 & 0 & \ldots & 0 & 0 & 0 \\
\frac{1}{n} & \frac{n-1}{n} & 0 & \cdots & 0 & 0 & 0 \\
0 & \frac{2}{n} & \frac{n-2}{n} & \cdots & 0 & 0 & 0 \\
\vdots & \vdots & \vdots & & \vdots & \vdots & \vdots \\
0 & 0 & 0 & \cdots & \frac{m-2}{n} & \frac{n-(m-2)}{n} & 0 \\
0 & 0 & 0 & \cdots & 0 & \frac{m-1}{n} & \frac{n-(m-1)}{n}
\end{array}\right)
$$

and $Q^{l}=\left(q_{0}^{l}, q_{1}^{l}, \ldots, q_{m-1}^{l}\right)^{\top}$ and $B^{l}=\left(b_{0}, b_{1}, \ldots, b_{m-1}\right)^{\top}$. The matrix form for $q_{i}^{r}$ is $M^{r} Q^{r}=B^{r}$, where $M^{r}=M^{l}$ and $Q^{r}=\left(q_{n-1}^{r}, q_{n-2}^{r}, \ldots, q_{m+1}^{r}, q_{m}^{r}\right)^{\top}$ and 
$B^{r}=\left(b_{n}, b_{n-1}, \ldots, b_{m+2}, b_{m+1}\right)^{\top}$. We obtain the following formulae for $q_{m}^{l}$ and $q_{m}^{r}$ from these systems by Gaussian elimination:

$$
\begin{aligned}
q_{m-1}^{l} & =\frac{1}{\left(\begin{array}{c}
n-1 \\
m
\end{array}\right)} \sum_{i=0}^{m-1}(-1)^{m-1+i}\left(\begin{array}{l}
n \\
i
\end{array}\right) b_{i}, \\
q_{m}^{r} & =\frac{1}{\left(\begin{array}{c}
n-1 \\
m
\end{array}\right)} \sum_{i=m+1}^{n}(-1)^{m-1+i}\left(\begin{array}{c}
n \\
i
\end{array}\right) b_{i} .
\end{aligned}
$$

Thus we obtain

$$
b_{m}-\frac{q_{m-1}^{l}-q_{m}^{r}}{2}=\frac{1}{2} \frac{1}{\left(\begin{array}{c}
n-1 \\
m
\end{array}\right)} \sum_{i=0}^{n}(-1)^{m+i}\left(\begin{array}{c}
n \\
i
\end{array}\right) b_{i}
$$

that is,

$$
\begin{aligned}
\left|b_{m}{ }^{x}-\frac{q_{m-1}^{l}{ }^{x}-q_{m}^{r x}}{2}\right| & =\frac{1}{2} \frac{1}{\left(\begin{array}{c}
n-1 \\
m
\end{array}\right)}\left|\sum_{i=0}^{n}(-1)^{i}\left(\begin{array}{c}
n \\
i
\end{array}\right) b_{i}{ }^{x}\right|, \\
\left|b_{m}{ }^{y}-\frac{q_{m-1}^{l}{ }^{y}-q_{m}^{r y}}{2}\right| & =\frac{1}{2} \frac{1}{\left(\begin{array}{c}
n-1 \\
m
\end{array}\right)}\left|\sum_{i=0}^{n}(-1)^{i}\left(\begin{array}{c}
n \\
i
\end{array}\right) b_{i}{ }^{y}\right| .
\end{aligned}
$$

REMARK 4. From (3.4), (3.5), (3.9) and (3.10), the $n$th degree Bézier curve $b^{n}$ is essentially the $(n-1)$ st degree Bézier curve if and only if

$$
\sum_{i=0}^{n}(-1)^{i}\left(\begin{array}{c}
n \\
i
\end{array}\right) b_{i}=0
$$

To compute $d\left(c_{m}, \frac{r_{m-1}^{\prime}+r_{m}^{r}}{2}\right)$, we first consider $\left|c_{m}{ }^{x}-\frac{r_{m-1}^{\prime}{ }^{x}+r_{m}^{\prime} x}{2}\right|$. From (3.9),

$$
\left|c_{m}^{x}-\frac{r_{m-1}^{\prime}{ }^{x}+r_{m}^{r x}}{2}\right|=\frac{1}{2} \frac{1}{\left(\begin{array}{c}
n-1 \\
m
\end{array}\right)}\left|\sum_{i=0}^{n}(-1)^{i}\left(\begin{array}{c}
n \\
i
\end{array}\right) c_{i}^{x}\right| .
$$

Then, from this formula and the subdivision formula (3.2) for $c_{i}$ at $c=\frac{1}{2}$,

$$
\begin{aligned}
\left|c_{m}{ }^{x}-\frac{r_{m-1}^{l}{ }^{x}+r_{m}^{r} x}{2}\right| & =\frac{1}{2} \frac{1}{\left(\begin{array}{c}
n-1 \\
m
\end{array}\right)}\left|\sum_{i=0}^{n}(-1)^{i} B_{i}^{n}\left(\frac{1}{2}\right) b_{i}{ }^{x}\right| \\
& =\frac{1}{2}\left(\frac{1}{2}\right)^{n} \frac{1}{\left(\begin{array}{c}
n-1 \\
m
\end{array}\right)}\left|\sum_{i=0}^{n}(-1)^{i}\left(\begin{array}{c}
n \\
i
\end{array}\right) b_{i}{ }^{x}\right|,
\end{aligned}
$$

that is,

$$
\left|c_{m}{ }^{x}-\frac{r_{m-1}^{l}{ }^{x}+r_{m}^{r x}}{2}\right|=\left(\frac{1}{2}\right)^{n}\left|b_{m}{ }^{x}-\frac{q_{m-1}^{l}{ }^{x}+q_{m}^{r x}}{2}\right|
$$




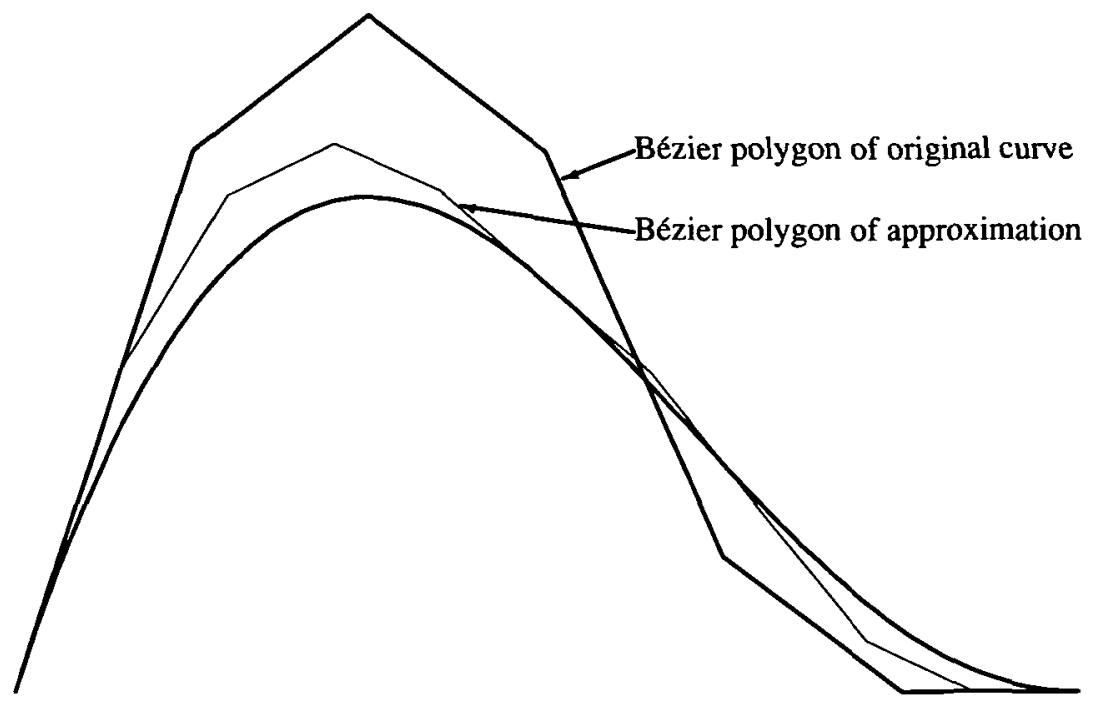

FIGURE 6. Degree reduction with 1-fold subdivision of a curve of degree 6.

We obtain the same form for $\left|c_{m}{ }^{y}-\frac{r_{m-1}^{t}{ }^{y}+r_{m}^{y}}{2}\right|$ by similar calculations. Combining these results, we obtain

$$
d\left(c_{m}, \frac{r_{m-1}^{l}+r_{m}^{r}}{2}\right)=\left(\frac{1}{2}\right)^{n} d\left(b_{m}, \frac{q_{m-1}^{l}+q_{m}^{r}}{2}\right) .
$$

Thus the approximation error $\left\|\left|e^{(1)} \|\right| \mid\right.$ after the first subdivision is

$$
\left\|\mid e^{(1)}\right\| \| \leq\left(\frac{1}{2}\right)^{n} \alpha_{n} d\left(b_{m}, \frac{q_{m-1}^{l}+q_{l}^{r}}{2}\right) .
$$

By induction on $k$, we reach the theorem:

THEOREM 5. The approximation error $\left\|e^{(k)}\right\| \mid$ after the kth subdivision is

$$
\left\|e^{(k)}\right\| \| \leq\left(\frac{1}{2}\right)^{n k} \alpha_{n} d\left(b_{m}, \frac{q_{m-1}^{l}+q_{l}^{r}}{2}\right)
$$

for even $n$, where $\alpha_{n}$ is

$$
2 \frac{1}{\left(\begin{array}{c}
n-1 \\
m
\end{array}\right)}\left(\frac{1}{2}\right)^{n}
$$




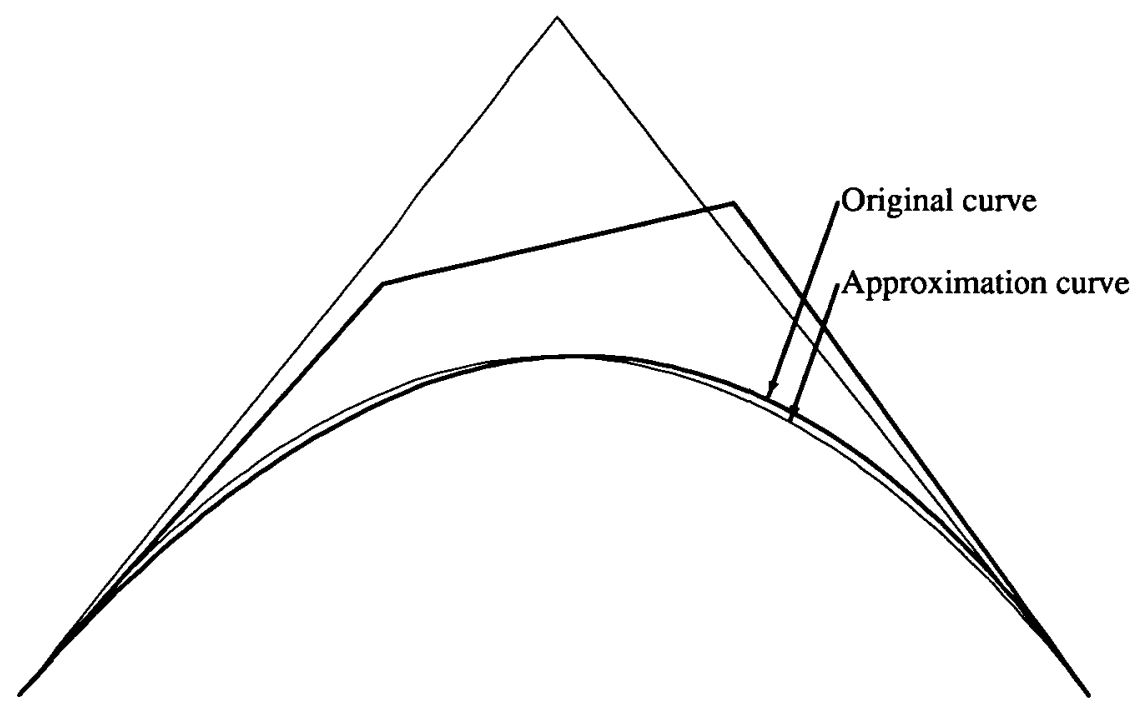

FIGURE 7. Degree reduction on a cubic Bézier curve.

Thus to obtain a piecewise Bézier approximation curve of degree $n-1$ to a given Bézier curve of degree $n$ to within a tolerance $\epsilon$, the number of subdivisions $k$ required is

$$
k \geq \frac{1}{n} \log _{2} \frac{\alpha_{n} d\left(b_{m}, \frac{q_{m-1}^{\prime}+q_{i}^{\prime}}{2}\right)}{\epsilon} .
$$

Figure 6 shows the result of applying the subdivision and degree reduction for even degree curve.

Table 1 shows the number of subdivisions and the maximum of the error in the approximating curves for a cubic curve in Figure 7. Figure 8 shows the error in the approximations.

TABLE 1. $k$-fold subdivisions and the maximum of the error.

\begin{tabular}{|c||c|}
\hline No. of Subdivisions & Maximum of the error \\
\hline 0 & $2.8900 \times 10^{-2}$ \\
\hline 1 & $3.6000 \times 10^{-3}$ \\
\hline 2 & $5.0000 \times 10^{-4}$ \\
\hline
\end{tabular}




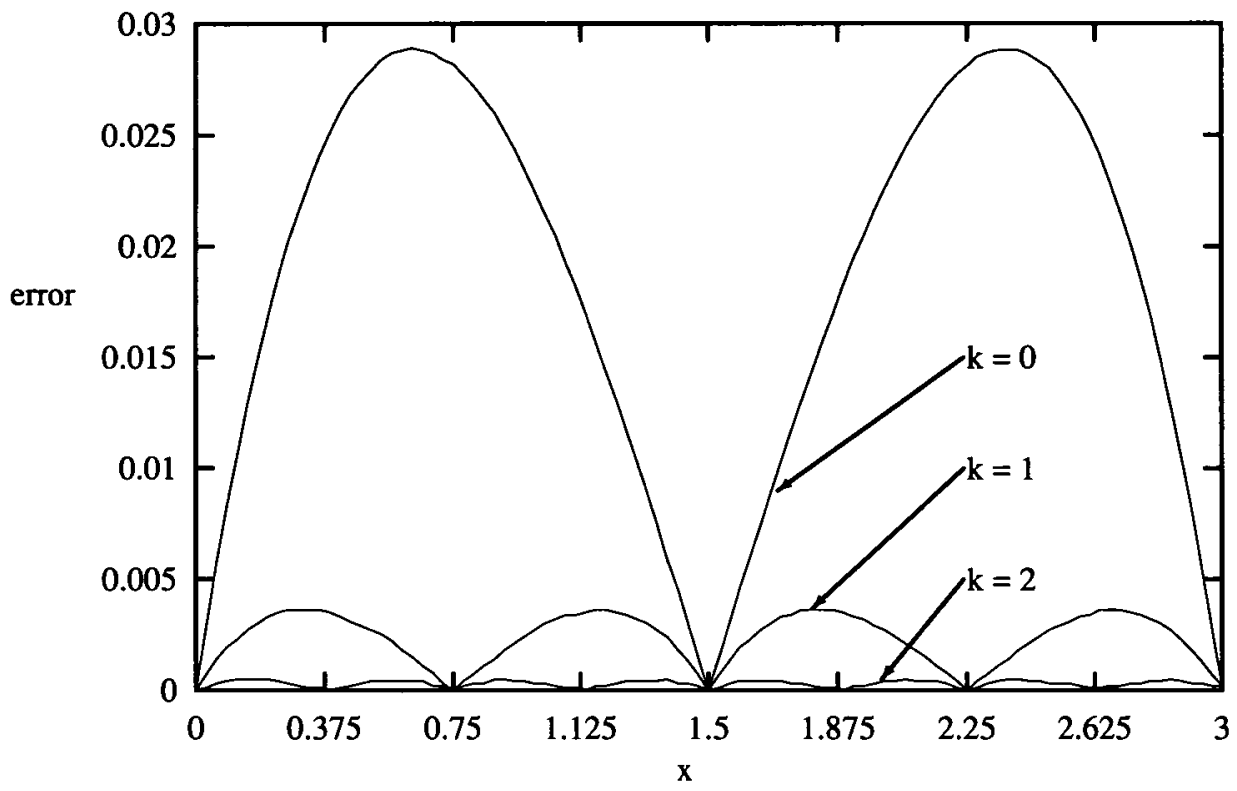

FIGURE 8. The error in the approximation.

\section{Acknowledgement}

This work was partially supported by KOSEF under Grant 91-08-00-01.

\section{References}

[1] W. Boehm, G. Farin and J. Kahmann, "A survey of curve and surface methods in cagd", Comput. Aided Geometric Des. 1 (1984) 1-60.

[2] L. Dannenberg and H. Nowacki, "Approximate conversion of surface representations with polynomial bases", Comput. Aided Geometric Des. 2 (1985) 123-131.

[3] G. Farin, "Algorithms for rational Bézier curves", Comput. Aided Des. 15 (1983) 73-77.

[4] G. Farin, Curves and surfaces for computer aided geometric design (Academic Press, New York, 1988).

[5] R. G. Hersch, "Font rasterization: the state of the art", 1991.

[6] J. Hoscheck, “Approximate conversion of spline curves", Comput. Aided Geometric Des. 4 (1987) 59-66.

[7] C. S. Petersen, "Adaptive contouring of three-dimensional surfaces", Comput. Aided Geometric Des. 1 (1984) 61-72.

[8] T. W. Sederberg and M. Kakimoto, "Approximating rational curves using polynomial curves", in NURBS for Curve and Surface Design (ed. G. Farin), (SIAM, Philadelphia, 1991) 149-158.

[9] M. A. Watkins and J. A. Worsey, "Degree reduction of Bézier curves", Comput. Aided Des. 20 (1988) 398-405. 